\title{
REPORT ON MATHEMATICAL INSTRUCTION IN CANADIAN UNIVERSITIES
}

Present: Professors H. I. Schiff (McGill)

Nominated by the Chemical Institute of Canada E. R. Pounder (McGill), G. M. Volkoff (B. C.) Nominated by the Canadian Association of Physicists A. Gauthier (Montreal), R. L. Jeffery* (Acadia), G. de B. Robinson (Toronto), M. Wyman (Alberta) Nominated by the Canadian Mathematical Congress

1. Background. At the meeting of the International Mathematical Union in Sweden last summer the Report of the International Commission on Mathematical Instruction made it clear that certain difficulties are arising throughout the world between departments of mathematics, physics and chemistry. The story of what is being done elsewhere to meet this situation stimulated some of us to organize the piesent meeting in the hope that we might be able to suggest remedies applicable in our Canadian Universities.

Prior to our meeting the accompanying article by Weinstock was circulated to members of the group and the following comments were received:

(a) Rigour is over-stressed in courses in mathematics attended by students of physics and chemistry,

(b) Mathematicians are unwilling to meet the needs of scientists for more advanced courses.

No disagreement with the se comments or with the position adopted by Weinstock was expressed by any member of the group. It was agreed that more cooperation is needed. How to achieve this cooperation was the subject of lengthy discussion. In the experience of those present however, there appeared to be one chief source of difficulty.

2. The choice of his major subject by an honours student may be too long delayed. In order to summarize the discussion on this point it should be emphasized that students come to Canadian Universities at two distinct levels. In some institutions they take a first course in the Calculus in their first year (e.g. Alberta, Toronto), in others in their second year (e.g. B. C., McGill, Montreal). For clarity, let us call

* Member of the International Commission on Mathematical Instruction 
the year in question the common introductory year. All of us agreed that such a common year should exist for honour students and we shall discuss its mathematical content in $\S \S 3,4$ below, but we were also agreed that to offer mathematical courses to students of mathematics, physics and chemistry, together beyond this common year may force the lecturer to compromise the rigour required by the professional mathematician for the benefit of those seeking a working knowledge of the subject. Whatever compromise is reached, the result may be unsatisfactory to all concerned. Thus we would recommend that students state their major interest at the end of their common introductory year and that subsequent courses in mathematics be arranged to meet the needs of each group.

3. Mathematical courses suitable to the common introductory year. We would suggest that two courses are appropriate, one in calculus and one in algebra and geometry (see $\S 4$ ), though it may be convenient to give the course in algebra and geometry in the following year.

With regard to the se two courses, we would suggest that the physicists and chemists should think carefully what specific techniques they actually require so that the mathematicians may plan the courses. It should be realized that to develop a subject properly certain time intervals are necessary. Thus these courses should be integrated with the appropriate courses in physics and chemistry so that each lecturer may know when the significant ideas with which he is concerned will be treated by his colleagues. This requires continuous cooperation throughout the year.

It was suggested that joint problems might be prepared at crucial points, but it was pointed out that if the mathematician reaches certain topics at known times and his colleagues know and use the same language in referring to mathematical concepts, it may be no disadvantage to have applications made by physicists and chemists independently. Some repetition is desirable at this early stage.

It was emphasized that much of the difficulty in this common introductory year arises through the inability of young lecturers to recognize the importance of the intuitive approach. Rigour should follow familiarity not precede it, even for students planning to become professional mathematicians.

4. Further discussion of this common introductory year emphasized the fact that modern physicists and chemists are utilizing vectors, matrices and groups almost as soon as the calculus, so that two introductory courses are really involved. Both these courses 
should be given by the mathematics department but in close cooperation with the departments of physics and chemistry. Mathematicians should remember that the origin of most mathematical ideas is physical and geometrical, and intuition springs from this common ground. On the other hand, physicists and chemists should recognize the continually increasing abstraction necessary to describe adequately natural phenomena. Students must have some practice in abstraction if they are to do more than manipulate formulae; this is why the trained mathematician is essential to the progress of science. Abstraction however, should not be confused with rigour; it is the method used to isolate certain a spects of reality common to many contexts. The physicists and chemists are seeking the same goal in this respect and need the language of mathematics to pass from the particular to the general.

5. Discussion of the common introductory courses in mathematics raised the question of the High School curriculum. The group was unanimously of the opinion that a conservative approach benefits the greatest number of students. Certainly, changes should be made, but to introduce ideas which cannot be applied by the student or a rigorous form of language useful only to a few is frustrating and useless. To a large extent these comments apply to the common introductory courses at the University as well. The notation of the calculus can cause confusion if not properly developed and explained, but it has proved a marvellous tool for 250 years and its value should be exploited from every point of view. The se introductory courses should provide the student with a well developed technique and an understanding of its use, as well as a knowledge of the pitfalls to be expected and avoided.

6. Courses beyond the common level will vary from university to university, since they should depend to a large extent on local needs and emphases. In those universities where theoretical physics or applied mathematics is recognized in the Calendar, common ground may exist even for two more years. In such cases it is desirable for the student to study mathematical models as well as mathematical methods in close conjunction. In some universities such courses are offered in the physics department and in others in the mathematics department. Personalities and histcrical patterns of development enter here and we can only stress the need for understanding and cooperation at the se higher levels also.

In this connection we would recommend that a sufficient number of mathematical options at the higher level be available for students of physics and chemistry. Since it is undesirable that courses on special functions, orthogonal expansions etc. be sandwiched into courses on theoretical physics, we would suggest that a half year 
course devoted to the appropriate mathematics might precede a course in its applications for such students, and a more rigorous course in analysis for students of mathematics.

7. Mathematics in biology, economics, psychology etc. Finally, we would make a plea for the teaching of mathematics in biology, economics, psychology etc. to be done by persons trained in the appropriate mathematical field. Abstraction and model building are becoming increasingly important in these disciplines also but Canadian universities have been only too ready to minimize their significance. In particular, the advent of the electronic computer has made it possible to handle statistics in a manner previously out of the question. The need for cooperation here is just as pressing, if universities are to prepare their students for graduate work elsewhere.
H. I. Schiff
A. Gauthier
E. R. Pounder
R. L. Jeffery
G. M. Volkoff
M. Wyman
G. de B. Robinson (Chairman) 